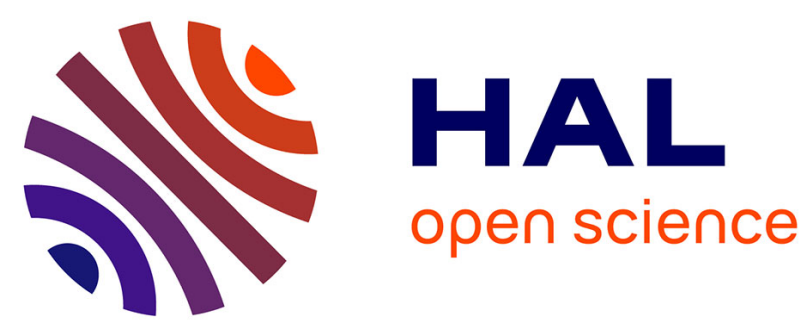

\title{
UNE SALLE DE CONCERT TRANSFORMABLE PAR VARIATION DU VOLUME EN OPÉRA : LA SALLE BERLIOZ DU CORUM DE MONTPELLIER
}

\author{
N. Auletta, D. Commins, C. Leneutre
}

\section{- To cite this version:}

N. Auletta, D. Commins, C. Leneutre. UNE SALLE DE CONCERT TRANSFORMABLE PAR VARIATION DU VOLUME EN OPÉRA : LA SALLE BERLIOZ DU CORUM DE MONTPELLIER. Journal de Physique IV Proceedings, 1992, 02 (C1), pp.C1-159-C1-162. 10.1051/jp4:1992131 . jpa00251202

HAL Id: jpa-00251202

https://hal.science/jpa-00251202

Submitted on 1 Jan 1992

HAL is a multi-disciplinary open access archive for the deposit and dissemination of scientific research documents, whether they are published or not. The documents may come from teaching and research institutions in France or abroad, or from public or private research centers.
L'archive ouverte pluridisciplinaire HAL, est destinée au dépôt et à la diffusion de documents scientifiques de niveau recherche, publiés ou non, émanant des établissements d'enseignement et de recherche français ou étrangers, des laboratoires publics ou privés. 


\title{
UNE SALLE DE CONCERT TRANSFORMABLE PAR VARIATION DU VOLUME EN OPERA : LA SALLE BERLIOZ DU CORUM DE MONTPELLIER
}

\author{
N. AULETTA, D.E. COMMINS et C. LENEUTRE* \\ 33 rue des Petits-Ruisseaux, BP. 81, F-91371 Verrières le Buisson cedex, France \\ *Chateau Descas, 3 bis quai de Paludate, F-33000 Bordeaux, France
}

\begin{abstract}
UNE SALE DE CONCERT TRANSFORMABLE PAR VARLATON DU VOLUME EN OPERA : LA SAUE BERUOZ DU CORUM DE MONTPEUER

RESUME :

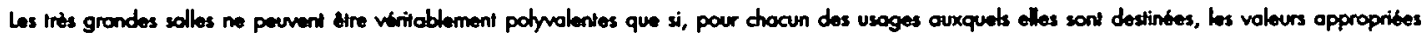

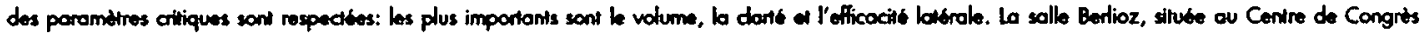
"le Conm" de le valle de Montpellier, est une salle dont le volume varie par une trandation verticale du plafond, qui permet d'exchure un niveaux de loges pour l'optra. Ces iransormotions morphotogiques permetient do respier les contraintes acoustiques dans doux cas :

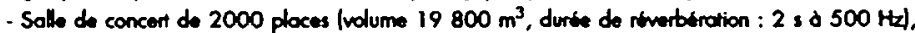

- Optra de 1815 ploces (volume $13600 \mathrm{~m}^{3}$, durfe de revertiotion : 1,5 s d $500 \mathrm{~Hz}$ ).

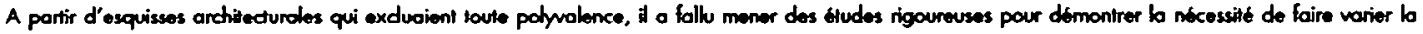

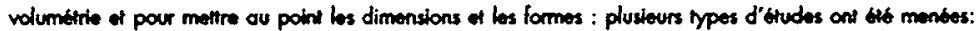

10) L'analyse des plans seton lo theorib sotigtique du champ diffus of celle de l'ocoustique geombtrique. a permis de mansmeltre aux archiledes les contraintes ocoustiques mojoures du projet (uolume, formes et trailements des surtaces de la selte, etc.).

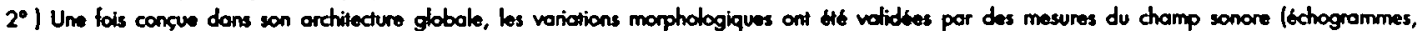

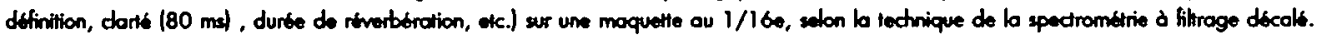

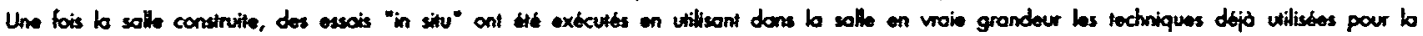
maquette. Les ritsulfals montrent que les objectifs on bib alteints a condition que la configuration choisie pour la salle corresponde au type de spectade

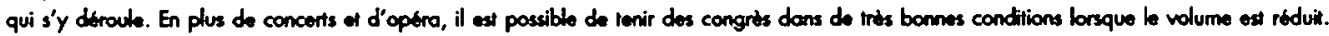

\section{A CONCERT MALL WHICH TRANSFOBMS INTO AN OPERA HOUSE THROUGH A VOWME OHANCE : BERUOZ HALl DN MONTPELUER} ABSTRACT :

Very large halls can not be truly multi-punpose untess, for exch of their uses, the appropriote values of all critical panametions are mot: the most inportant ore volume, dority and lateral efficioncy. The volume of Bertioz Hall, which is part of the "Conum" cutural complex in Montpellier, can be modified by a vertical translation of the ceiling, thus axduding two levels of boxes. Thase morphological transformations mate the hall acoustically compatible with two moin uses:

- Symphony holl of 2000 seats (volume: $19800 \mathrm{~m}^{3}$, rewerberation tima: 2 s d $500 \mathrm{~Hz}$ )

- Opera House of 1815 soots frolume: $13600 \mathrm{~m}^{3}$, reverberation time: 1,5 i d $500 \mathrm{~Hz}$ ).

Starting from archifectural sketches which excluded any flexibitity, rigorous investigations hove boen conducted to demonstrote the noed for a variable votume and to dotine dimensions and shopes. Several lectriques hove been used :

1) An analysis of drowings on the basis of the statistical analysis of diffused fiolds and on the basis of acoustical geomatry has led to the definition of the major paramolers: volume, shope, materials, etc.

2) Once the averoll anchinecture had been set, morphological changes have been validated by acoustic lests on a 1/16 seale model using lime-doloy spectrometry limpulse response, iniolligiblity, darity, reverberation time, otc.).

Afier complotion of the holl, on site meosurements hove been pertormed, whit the same lectnique. They hove shown that the design gools hod been reached. This hes been widely confirmed by musicians and spoctators alike. In addition to concents and operas, congreswes can take ploce in the hall under good conditions in the "opero" configuration. 


\section{INTRODUCTION}

Les programmes archilecturoux des salles prévoient très souvent des octivités en principe incompatibles entre elles. Le cas de salles de petite capacité, c'est à dire de moins de 600 places est relativement aisé à résoudre. Les très grandes

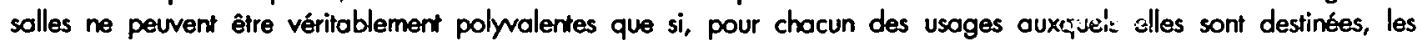
valeurs appropriées des paramètres critiques sont respectées. Les plus importants sont : le rilins, ia clarté et l'efficacité latérale.

Trois éléments permettent de moduler l'acoustique interne d'une salle :

- la morphologie générale et la forme des composants,

- I'habillage des parois, lorsque le volume optimum par spectateur est similaire (ex.: théâtre et conférence)

- le volume, si le volume optimum par spectateur diffère (ex.: concert el opéra).

Le programme de la salle Berlioz, située au Centre de Congrès "le Corum" de la Ville de Montpellier, donnait la priorité aux concerts symphoniques mais donnait une grande place à l'opéra et aux congrès. L'option a donc été prise de laire varier le volume par une translation verticale du plafond, qui permel d'exclure un niveau de loges pour l'opéro. Les congrès se déroulent dans de bonnes conditions dans cette dernière configuration.

\section{LA CONCEPTION DE LA SALLE}

\subsection{Etude de la salle por la théorie de Sabine}

La théorie de Sabine est un modèle qui se vérifie assez bien dans une salle de concert, par les objectifs conceptuels qu'elle propose :

- La densité d'énergie sonore doit être la plus homogène possible,

- la relation entre la longueur, la largeur, et la hauteur doit être inférieure d̀ 2, quelle que soit la combinaison entre elles.

- la seule hypothèse de la théorie de Sabine qu'une salle de concert ne vérifie pas est la distribution uniforme des matériaux absorbants sur toutes les surfaces : dans une salle de concert, en effet, l'unique surface absorbante est celle occupe par te public (ò sovoir, le plancher ainsi qu'une partie variable des parois latérales).

Les dimensions moyennes de la salle Berlioz (configurée opéra) sont : longueur $31,9 \mathrm{~m}$, largeur $28,5 \mathrm{~m}$, hauteur $15 \mathrm{~m}$. Dans cette configuration, le volume de salle (fosse comprise et scène excluel est $V_{o p}=13620 \mathrm{~m}^{3}$; la durée de réverbération, aux fréquences moyennes (octoves centrées à $500 \mathrm{~Hz}$ et $1 \mathrm{kHz}$ ) est $T_{\text {op }}=1,5 \mathrm{~s}$.

Les dimensions de la salle ont été fixées en considérant le nombre de spectateurs (1815 en configuration opéra) ainsi que, pour les zones les plus éloignées de la scène, le fait que la densité sonore directe ajoutée aux réflexions premières ne soit pas inférieure à la densité d'énergie sonore réverbérée. Celte condition est liée à l'intelligibilité du livret.

Les dimensions requises par une salle d'opéra élant fixées, il fallait ensuite adapter le volume afin que l'on puisse, d'une part, accueillir 2000 personnes en concert et, d'autre part, obtenir une durée de réverbération de 2 s., pour les octaves centrées aux fréquences de $500 \mathrm{~Hz}$ ef $1 \mathrm{kHz}$.

A partir de la théorie de Sabine et des contraintes fixées, l'augmentation de volume est donnée

$$
1+\frac{\Delta T}{T_{o p}}=\left[1+\frac{\Delta V}{V_{o p}}\right]\left[1+\frac{\Delta A}{A_{o p}}\right]^{-1}(1) \quad A_{o p}=\text { aire d'absorption totale de la salle en configuration opéra. }
$$

L'équation (1) permet de tirer la conclusion suivante : pour que corresponde ò une augmentation du volume une augmentation de la durée de réverbération, il faut que : $\frac{\Delta A}{A_{o p}} \ll<1 \Rightarrow \Delta V>\frac{\Delta T}{T_{o p}} \times V_{o p}$

Alors, les surfaces ajoutées doivent être réfléchissantes et diffusantes, dans la mesure du possible.

Les dimensions horizontales moyennes de la salle dans sa configuration concert sont identiques à celles de la configuration opéra : seule la hauteur est augmentée de $6 \mathrm{~m}$ environ. A celte augmentation du volume de la salle, il faut ajouter celle de la conque d'orchestre placée sur scène, d'approximativement $1650 \mathrm{~m}^{3}$ /configuration symphonique ovec choeur) (Cf. Figures la et lb).

Celte étude, à partir de la théorie de Sabine, de l'application des lois de la réflexion de la théorie géométrique et de la définition de critères a permis de fixer :

- le volume de la salle

- le positionnement des parois

- le type de traitement de ces parois : réfléchissantes, diffusontes et absorbantes.

Ainsi, à la fin de la phase d'étude détaillée du projet, la solle fut définie dans so quasi-totalité en ce qui concerne l'acoustique, l'architecture et la scénographie. 


\subsection{Etude sur maquette de l'acoustique interne de la salle}

Considérant l'importance de l'ouvrage ef du fait qu'aucune étude théorique basée sur une approche linéaire ou statistique du champ sonore ne représente pas la réalité ondulatoire de celui-ci, une étude surs maquette au $1 / 16 e$ a été réalisée.

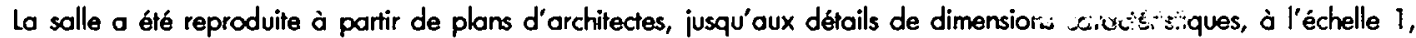
d'approximativement $0,5 \mathrm{~m}$, sauf pour les inclinaisons des parois ou variations de rivitivi: pour lesquelles des dimensions jusqu'd̀ $0,15 \mathrm{~m}$ ont été prises en compte.

Le public a été représenté par une surface absorbante. Quant à son volume, lequel ajoute au champ sonore de la diffusion et de la diffraction en plus de l'absorption, il n'a pas été modélisé.

L'obję des essais sur maquette a donc áfé de vérifier l'acoustique interne de la salle pour les couples émission/réception afin de connaître :

- la réponse impulsionnelle (forme, possibilité d'échos)

- la distribution du champ sonore

- la bolance énergétique entre l'énergie précoce et l'énergie réverbérée

- la clarté et la définition

- la vérification de la variation relative de la durée de réverbération en configuration concert el opéra due à la modification du volume de la salle.

Les mesures ont été faites par la technique de la spectrométrie à filtrage décalé, laquelle consiste à émettre un signal sinusoïdal glissant de courte durée, soit dans la conque (configuration concert), soit sur la scène ou dans la fosse (configuration opéra). La réponse acoustique de la salle a été mesurée pour l'octave de $8 \mathrm{kHz}$ (500 Hz à l'échelle 1) à l'aide d'un microphone placé à $80 \mathrm{~mm}$.

Les figures 2 et 3 montrent lo réponse impulsionnelle de la salle dans les deux configurations.

\subsection{MESURES DE RECEPTION - ACOUSTIQUE INTERNE}

Les mesures réalisées après la construction du Corum pour caractériser l'acoustique interne de la salle Berlioz (salle vide) ont été de deux types:

- mesure de la réponse (concert el opéra) à un signal sinusoidal glissant de courte durée (octove figurée $250 \mathrm{~Hz}$ ).

- mesure classique de la durée de réverbération pour les octaves centrées entre $63 \mathrm{~Hz}$ el $8 \mathrm{kHz}$.

Les figures 4,5 et 6 montrent les réponses de la salle dans les deux configurations.

\section{CONCLUSIONS}

Les plus importantes conclusions que l'on puisse tirer de cette réalisation sont:

- Les études ont permis d'aboutir à une salle dont l'acoustique interne répond aux objectifs fixés, c'est-à-dire dont les paramètres acoustiques varient selon la configuration choisie, ainsi que le montre notamment la figure 6 pour la durée de réverbération. Les musiciens et les mélomanes l'ont d'ailleurs largement confirmé.

- La durée de réverbération de la salle Berlioz est, pour les octaves moyennes et aiguës, plus courte que celle prévue. Nous constatons là un problème déjà connu : la marge d'erreur due à l'extrapolation des valeurs des coefficients d'absorption mesurés en laboratoire à l'implantation du même matériau in situ. Ce problème est particulièrement sensible en ce qui concerne les fauteuils, ceux-ci occupant une surface très importante et leur implantation dans la salle étant différente à celle adoptée en général lors des essais en laboratoire.

- Les paramètres liés à la durée de réverbération, tels que la définition et la clarté (80 ms) ont, dans la salle Berlioz en configuration concert et opera, des valeurs positives.

Dans nos études sur maquette, ces paramètres entre configuration concert et opéra présentaient des écarts plus importants.

- Les échogrammes pris dans la salle présentent les mêmes caractéristiques que ceux mesurés sur la maquette.

C'est ou Professeur Cremer, qui a activement participé aux phases initiales du projet, que les auteurs doivent ta confiance qu'ils ont développée envers ce concept. lis lui en sont très reconnoissants. 

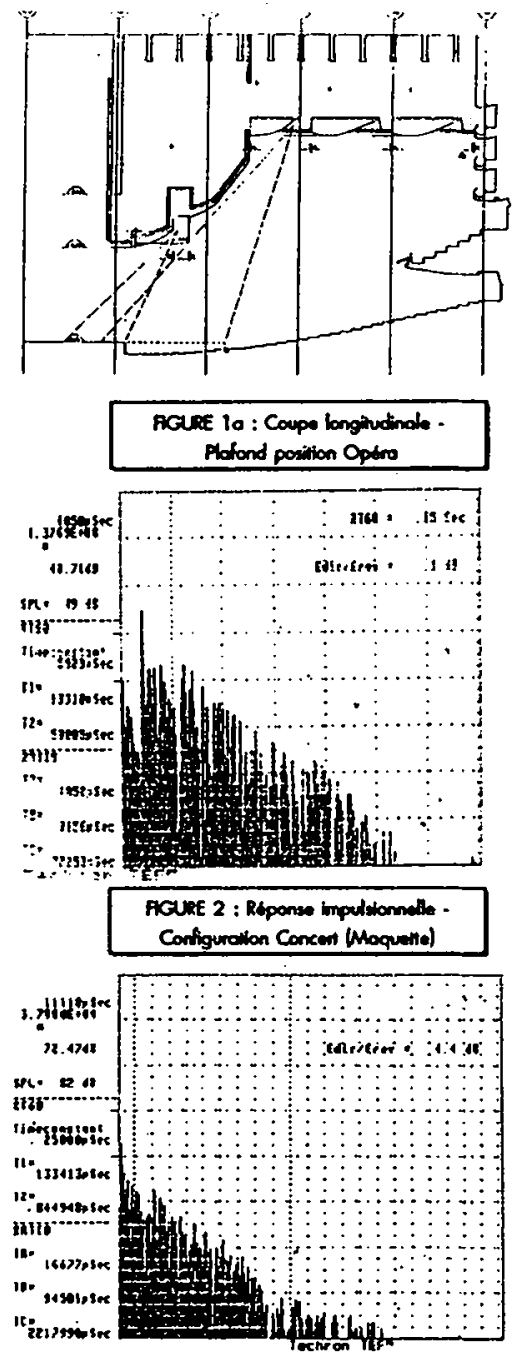

AGURE 4 : Reponse de b salle rieloen configuration Concent
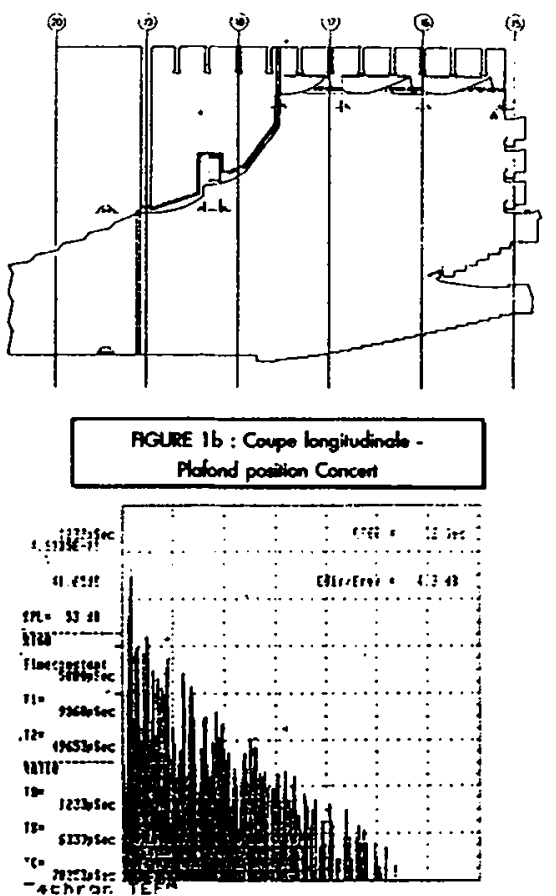

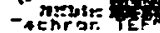

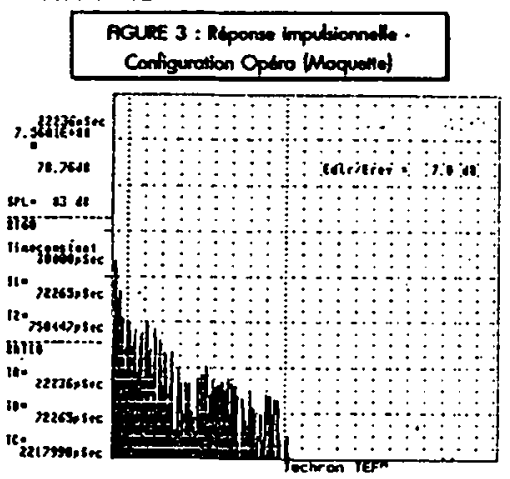

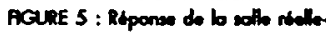
en configurction Optro

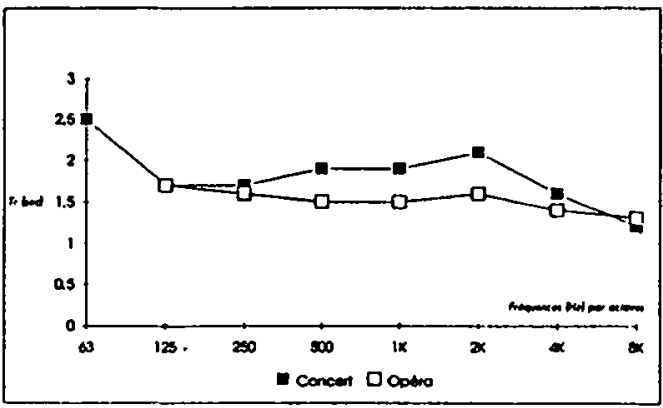

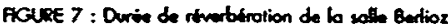
en configuration Concent et Optro 